\title{
The Role of B-Line Artifacts on Lung Ultrasound in Critically Ill Patients
}

\author{
Ana Pičuljan ${ }^{1, *}$, Alen Protić ${ }^{1}$, Mehmed Haznadar ${ }^{2}$, Alan Šustić $^{1,3}$
}

\author{
${ }^{1}$ Department of Anesthesiology, \\ Reanimatology, Intensive Care and \\ Emergency Medicine, Faculty of \\ Medicine, University of Rijeka, Croatia \\ ${ }^{2}$ Department of Anesthesiology, \\ Reanimatology and Intensive Care \\ Medicine, Cantonal Hospital Mostar, \\ Bosnia and Herzegovina \\ ${ }^{3}$ Department of Clinical Medical Science \\ II, Faculty of Health Studies, University \\ of Rijeka, Croatia \\ *Correspondence \\ ana.piculjan@student.uniri.hr \\ (Ana Pičuljan)
}

\begin{abstract}
Lung ultrasound (LUS) is becoming a valuable tool in assessing patients in the ER, ICU, and on the hospital wards because it is a simple, fast and harmless technique. One of the most distinct artefacts seen with LUS are B-lines, hyperechoic laser-like lines that form in the presence of thickened interlobular septa due to alveo-interstitial syndrome. Many researches have been conducted recently with a goal of correlating B-lines to specific known parameters such as EVLW, PAOP, lung density, and parameters of cardiac failure, either in quantitative or semiquantitative manner. This paper is a short overview of the present known correlations, shortcomings of conducted investigations and differential diagnosis problems and exceptions of B-line artefacts in everyday clinical practice.
\end{abstract}

\section{Keywords}

Point-of-care-ultrasound (POCUS), Lung ultrasound, B-lines

\section{Introduction}

Lung examination is essential in almost all acute or chronic states involving heart and lung pathology. Different methods of examination vary from physical examination, such as auscultation and percussion to imaging techniques, such as X-ray and computerized tomography (CT). Lung ultrasound (LUS) was initially considered suboptimal for lung examination as the lungs were deemed poorly suited to ultrasound beams that were dissipated by air media resulting in inadequate display of the lung parenchyma [1].

With a better understanding of LUS interpretation, this imaging modality has found more application in various clinical settings such as the ER, ICU, and medical wards. While LUS has a steep learning curve, advantages of this imaging modality include rapid examination times, reproducibility, and patient safety. At present, many studies are being performed using LUS, making correlations, and quantitative and semiquantitative estimates, of well-known parameters such as pulmonary artery occlusion pressure (PAOP), extravascular lung water (EVLW), or N-terminal pro-brain natriuretic peptide (NT-proBNP) possible.

Today, a huge range of terminology exists regarding various artifacts observed when conducting LUS. For example, Alines are horizontal reverberation artifacts that represent normal lung presentation. E-lines are vertical artifacts that arise from subcutaneous tissue and usually represent underlying subcutaneous emphysema. Z-lines are poorly-defined vertical artifacts and their significance is still not well understood $[2,3]$. Lung artifacts have been found to correlate with specific lung diseases or states (i.e., absence of lung sliding is highly indicative for pneumothorax, presence of B-lines suggests interstitial pathology). Other useful signs include the 'seashore' and 'stratosphere' signs used in the diagnosis of pneumothorax and the 'lung point' sign used to delineate the anatomic border of pneumothorax $[2,3]$.

B-lines are the most striking artifacts found with LUS, and are associated with alveo-interstitial syndrome. The most common encountered pathology of this syndrome is generally interstitial edema, but B-lines can be observed in other pathologies such as pulmonary fibrosis or interstitial pneumonia [46]. Many researchers are investigating the direct correlation between the number of B-lines, their localization, and pattern with findings obtained using other imaging methods (such as X-ray radiography and $\mathrm{CT}$ ), invasive hemodynamic monitoring, and neuro-hormonal parameters. This review covers the current literature concerning the use of LUS in everyday practice in the ER, ICU, and medical wards. Furthermore, we review available information regarding the definition of Blines and their use, various methods of thoracic examination, and some specific conditions in critically ill patients that correlate with B-line artifacts observed using LUS.

\section{Definition of B-lines}

B-lines are hyperechoic laser-like lines with a narrow base formed at the pleural line that reaches all the way to the bottom of the US screen and B-lines move synchronously with lung respiration [7]. In keeping with other US findings, B-lines are formed by a beam reflection at the interface of two areas of differing acoustic impedance - e.g., between the air-filled lung and thickened interlobular septa (by water or fibrous tissue). In normal circumstances, interlobular septa cannot be visualized by US because they are below the resolution of 


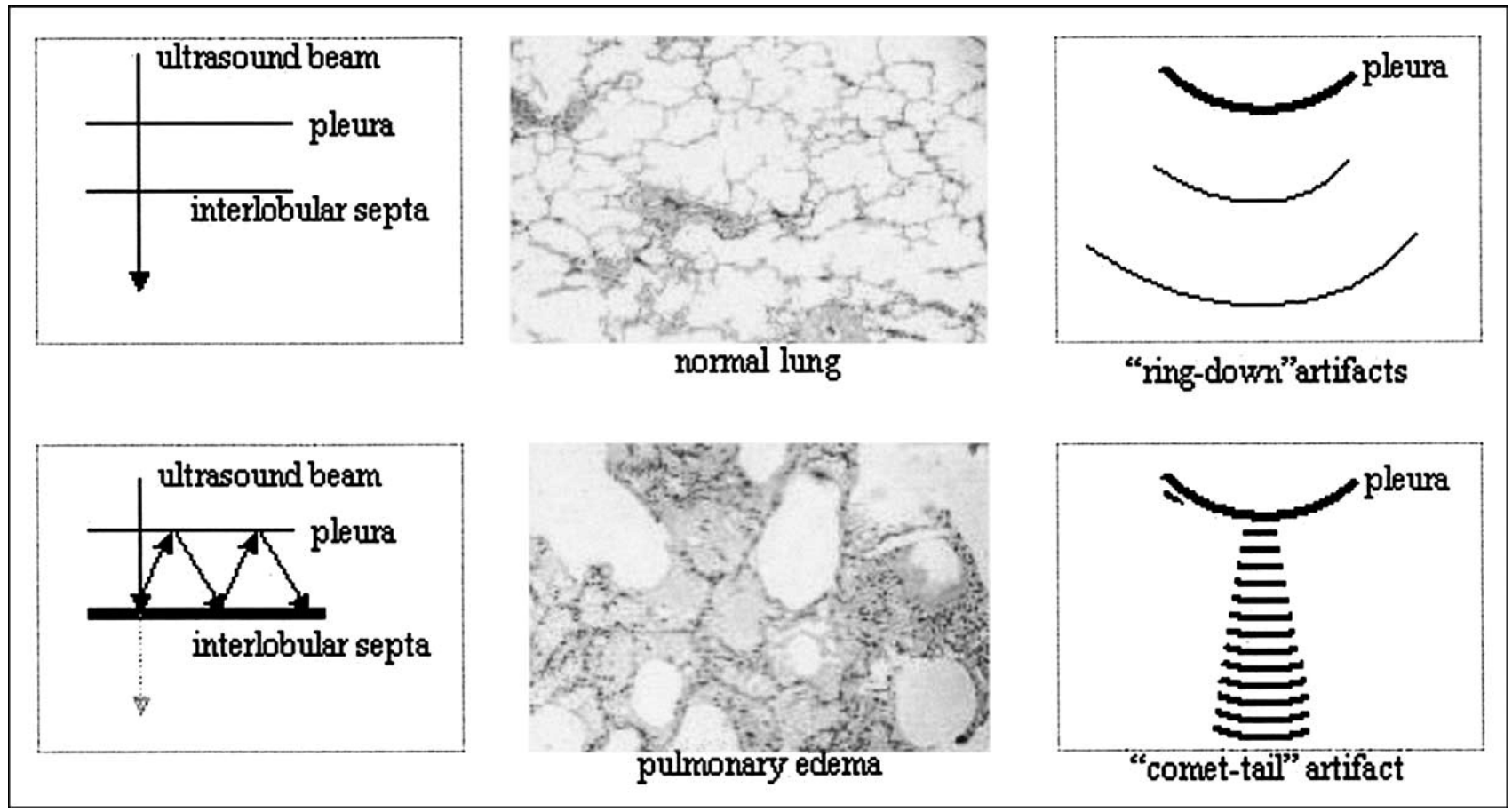

F I G U R E 1. Schematic image of B-line artifact formation by reverberation phenomenon.

Source: Jambrik Z, Monti S, Coppola V, Agricola E, Mottola G, Miniati M, et al. Usefulness of ultrasound lung comets as a nonradiologic sign of extravascular lung water. Am J Cardiol. 2004;93(10):1265-70. [8].

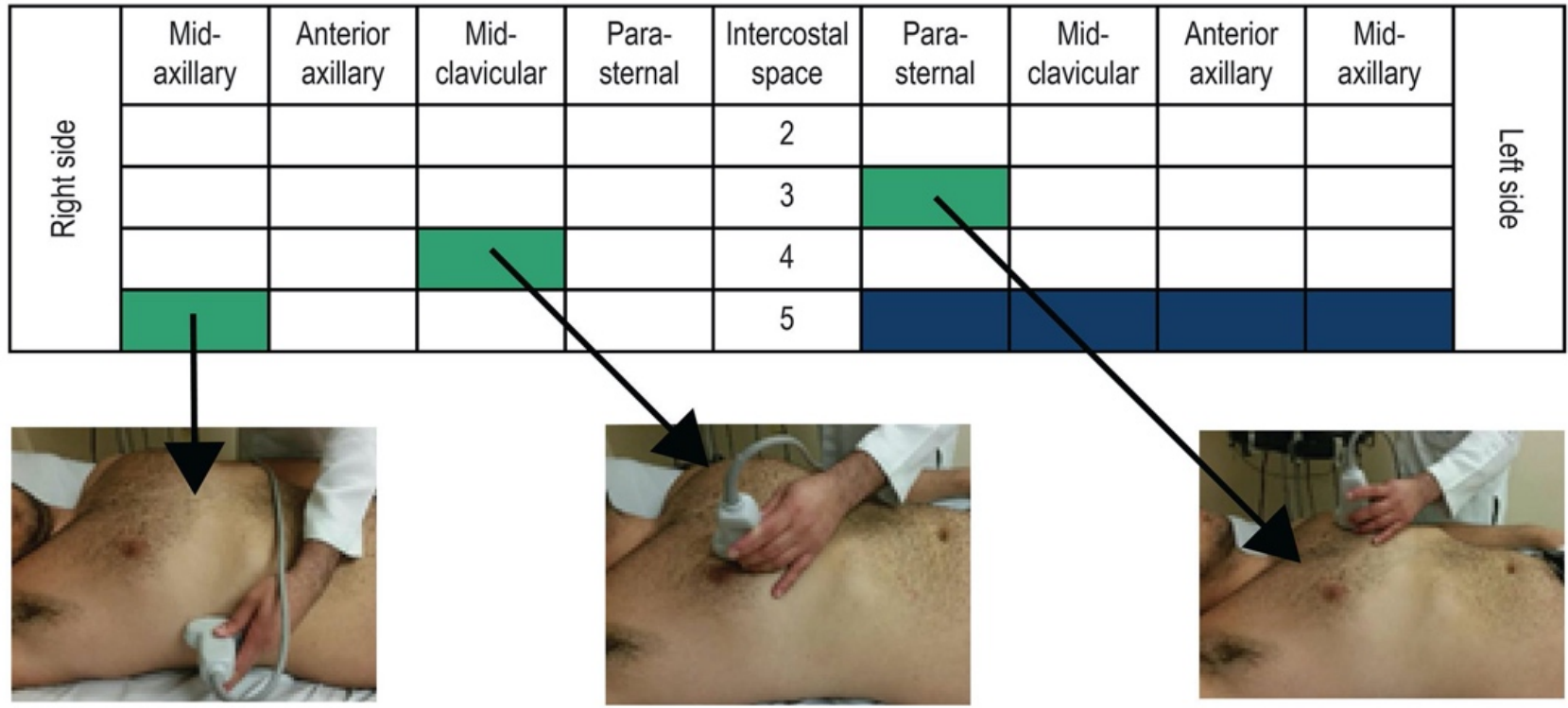

F I G U R E 2. Method of lung scanning via 28 scanning windows.

Source: Jambrik Z, Monti S, Coppola V, Agricola E, Mottola G, Miniati M, et al. Usefulness of ultrasound lung comets as a nonradiologic sign of extravascular lung water. Am J Cardiol. 2004;93(10):1265-70. [8].

the US beam; however, when alveolar-interstitial syndrome is present B-lines are visualized as a comet tail artifact. Blines are formed because of the continuous reflections of US beam from the interface of different areas. Specifically, there is a reverberation phenomenon observed as the reflections are continuously returning to the US probe (Fig. 1, [8]). As the US probe interprets time as distance, an image of a beam is created on the US screen $[4,7]$.

\section{Use of LUS B-lines}

The appeal of LUS in everyday clinical practice lies in the fact that it is fast, available at the bedside, safe and reproducible method which does not require patient transfer to CT $[4,7$, 9]. This makes point-of-care-ultrasound (POCUS) a valuable asset and an ideal tool for guiding therapy [4] Being a rapid and almost always available diagnostic method, LUS is very appealing in treating critically ill patients in whom the balance between volume optimization and volume overload is delicate, and where volume overload can easily lead to pulmonary 
edema and impaired gas exchange. In the primary evaluation of a patient, invasive methods for estimating hemodynamic status are not available, and one cannot rely on them for guidance in the initial treatment [10]. Even within the ICU setting, when more sophisticated methods are available, LUS is appealing as a fast and reproducible imaging modality that can be utilized for serial measurements and patient monitoring without exposing the patient to excessive radiation or the risks of transfer [4]. More recently, LUS has been shown to be useful for estimating lung aeration after antibiotic therapy of pneumonia, as well as for titrating positive end-expiratory pressure (PEEP) during lung recruitment [4]. Such uses make LUS an irreplaceable tool in the ICU.

B-lines have found application in numerous acute and chronic conditions that affect lung interstitium, and consequently alveo-capillary membrane gas exchange, causing respiratory distress. Conditions such as pneumothorax, acute lung edema, acute respiratory distress syndrome (ARDS), interstitial pneumonia, pleural effusion, or pulmonary fibrosis can be assessed by LUS. The correlation between B-lines and each of these conditions is being investigated, with B-lines being used for narrowing the differential diagnosis of acute respiratory failure due to the fact that the absence of B-lines can exclude interstitial syndrome [4, 7, 9]. In some conditions like ARDS and fat embolism, LUS detects B-lines even up to 2 - 3 days before the symptoms manifest. In early ARDS, LUS shows sensitivity of $98 \%$ and specificity of $88 \%$, which is superior in diagnosing interstitial syndrome to auscultation or X-ray radiography $[4,7]$.

\section{Thorax examination methods}

There are a few methods of scanning the thorax for B-lines, but the approach most frequently used is anterolateral scanning with the patient placed in a supine position. This approach consists of scanning the thorax in mid-axillary, anterior axillary, mid-clavicular and parasternal intercostal spaces, from the second to the fifth space on the right side, and from the second to the fourth space on the left side resulting in a total of 28 different scanning windows (Fig. 2, [7, 8]). Other described techniques include Volpicelli's 4-quadrant approach (Fig. 3, [6]) in which the hemithorax is divided into four quadrants with anterior axillary line in the vertical plane and second intercostal space in the horizontal plane. Four quadrants are scanned on each side of the thorax, and B-lines counted, thus resulting in a total sum of 8 windows [7]. Even though there are numerous protocols requiring 4 to 28 scanning sites, a 6 -site scan using the BLUE protocol (Fig. 4, [11]) has been found sufficient and time sparing for congestion detection when compared to other protocols [12].

\section{B-lines and pneumothorax}

Pneumothorax is a condition that demands rapid detection and intervention. It can be easily detected bedside using LUS with up to $85-100 \%$ sensitivity. In contrast, X-ray imaging has been shown to miss the diagnosis in approximately $30 \%$ of cases. Moreover, when we use the absence of B-lines and lung sliding together, sensitivity is $91 \%$ and specificity $98 \%$.
This is in contrast to X-ray imaging which has $50 \%$ sensitivity and $99 \%$ specificity [7]. Taken together, LUS is a superior approach to the diagnosis of pneumothorax compared to X-ray radiography.

Lung evaluation with LUS depends on the visualization of pleural membranes and on the reverberation of US beams along the contact area of visceral pleura and underlying alveolar tissue. The beam reverberating from the line of visceral pleura and representing the reflection of the pleural line makes an Apattern, a sign of 'dry lung'. The beam reverberating from the thickened interlobular septa makes a comet-tail artifact or a B-line, and is a sign of 'wet lung'. Normal lung has Apattern and some B-lines in lung bases along with the 'lung sliding' phenomenon which represents sliding of visceral and parietal pleura. Lung sliding is visualized as a flickering of the hyperechoic pleural line with movement excursions. Both of these reverberation phenomena require the presence of alveolar tissue underneath the pleural line, helpful in detection of pneumothorax, along with the absence of lung sliding. In pneumothorax cases the operator seeks to identify the 'lung point', where normal lung tissue in contact with pleura shares the same window with the air of pneumothorax underneath the pleura, either in 2D or in M-mode [9].

Although lung point was considered pathognomonic for pneumothorax, Aziz et al. presented a case where they found a lung point sign without the presence of underlying pneumothorax in a patient with bullous lung disease. The same substrate, air under pleura, produced a lung point sign on LUS, and was correctly diagnosed by CT scan. Caution should therefore be applied to the lung point sign found in patients with the history of bullous lung disease so that the unnecessary and potentially harmful interventions can be prevented [9]. It should also be noted that overlapping an unrecognized B-line with the cursorline in $\mathrm{M}$ mode during respiratory movements can produce a bar-code sign which mimics pneumothorax [13].

\section{B-lines, EVLW, and PAOP}

Extravascular lung water (EVLW) is a direct sign of lung congestion, with a usual cut off value of $10 \mathrm{ml} . \mathrm{kg}^{-1}$ being the threshold for acute lung injury. It is usually estimated by thermodilution invasive methods like Pulse Contour Cardiac Output (PiCCO). Pulmonary artery occlusion pressure (PAOP) estimates the pressure that spreads from pulmonary capillaries to the left ventricle and is a predictor of hemodynamic congestion/overload. The usual cut off value in clinical practice is $18 \mathrm{mmHg}$ [10]. Some studies have demonstrated a strong correlation between A-pattern and low PAOP. However, caution must be taken when predicting high PAOP as one must take into consideration that high values do not necessarily mean extravasation of fluid into the lung interstitium, and consequently, the appearance of B-lines. A higher threshold for developing lung edema with high PAOP was observed, for example, in patients with chronic heart failure. Also, low PAOP with high membrane permeability can result in noncardiogenic edema and B-pattern. Volpicelli et al. noticed that patients with high PAOP who showed A-pattern had chronic heart failure, and the combination of high PAOP and B-pattern was detected largely in patients with respiratory disease and 


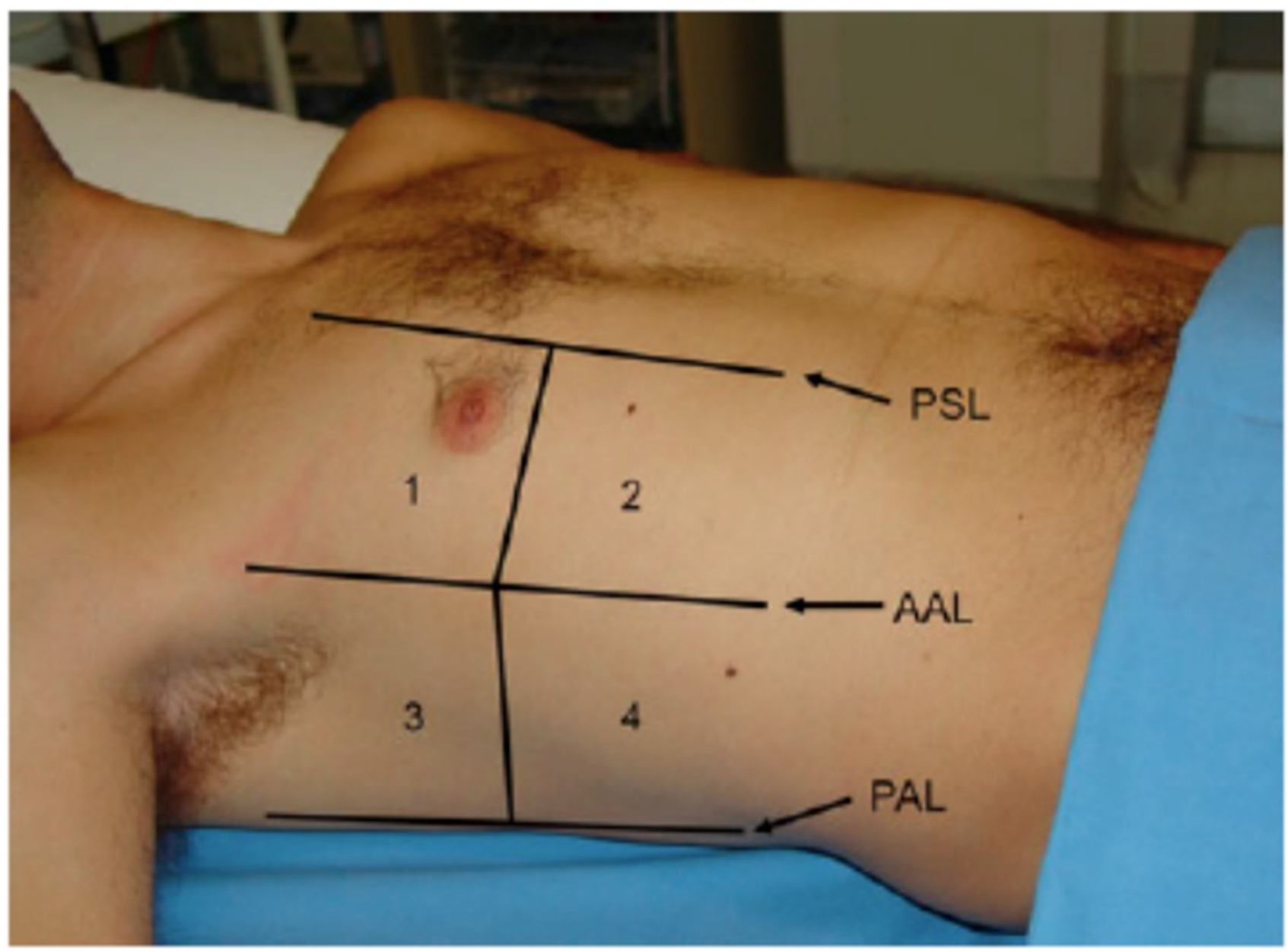

F I G U R E 3. Volpicelli's 4-quadrant approach.

Source: Volpicelli G, Mussa A, Garofalo G, Cardinale L, Casoli G, Perotto F, et al. Bedside lung ultrasound in the assessment of alveolar-interstitial syndrome. Am J Emerg Med. 2006;24(6):689-96. [6].

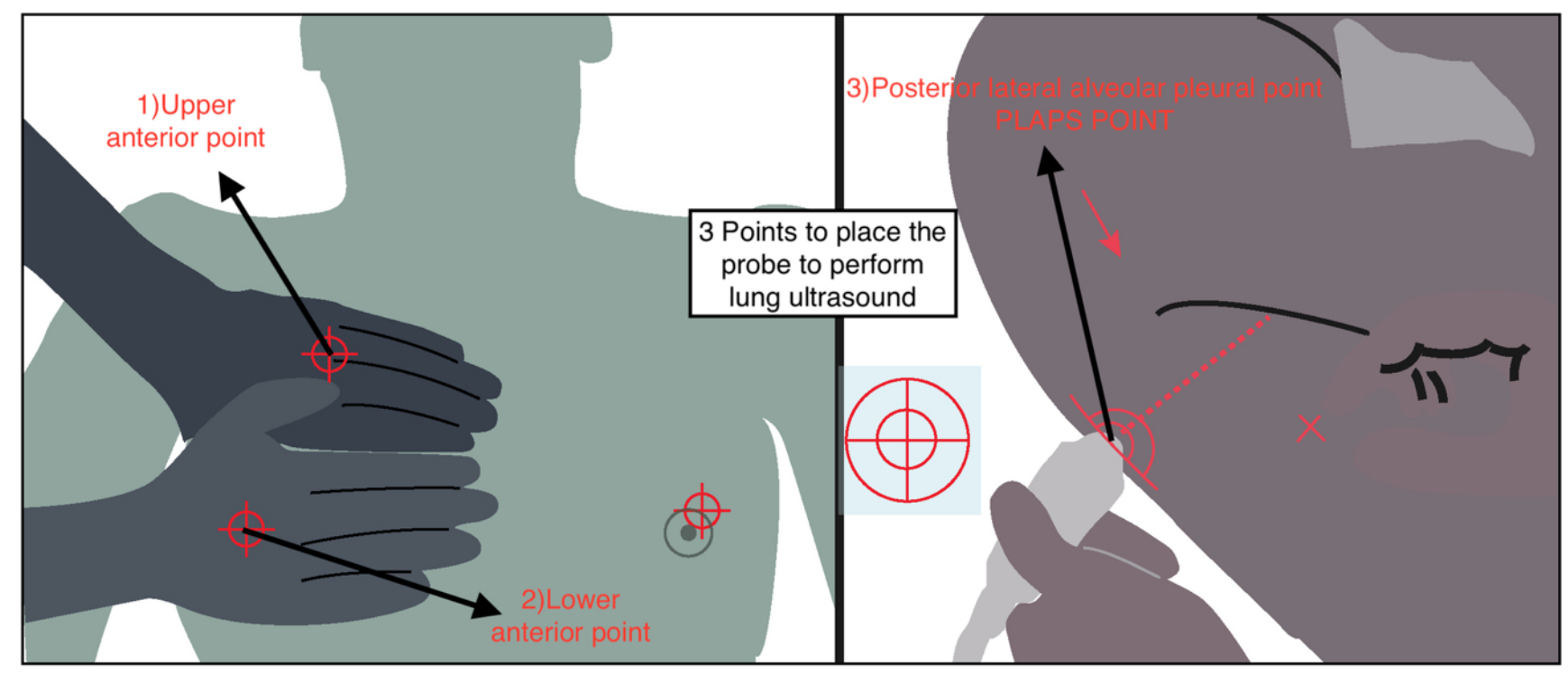

\section{F I G U R E 4. BLUE protocol scanning points.}

Source: Raheja R, Brahmavar M, Joshi D, Raman D. Application of Lung Ultrasound in Critical Care Setting: a Review. Cureus. 2019;11(7):e5233. [11]. 
sepsis, but with normal cardiac function [10]. These findings led the workers to suggest that PAOP and lung fluids are not necessarily connected [10]. In the Volpicelli et al. study, A and B-pattern in combination with normal or impaired LVEF were correlated with cut off values for PAOP and EVLW (Table 1). A good prediction for EVLW was observed, but there was limited prediction of PAOP. Of note, when B-pattern was combined with LVEF, the prediction of PAOP improved [10].

A-pattern predicts low EVLW, but cannot be used independently for predicting low PAOP. A low PAOP can be presumed only when A-pattern is combined with normal LVEF (i.e., > 55\%). B-pattern combined with impaired LVEF was highly predictive for high PAOP, which could be of use in differential diagnosis of ARDS. In conclusion, LUS can be used for excluding lung congestion, but in order to exclude hemodynamic congestion, a rough LVEF estimate must be performed as well. With this in mind, A-pattern in initial patient assessment indicates good fluid administration tolerance [10].

Volpicelli et al. intended to show only a qualitative correlation between B-pattern, EVLW and PAOP, with accent on rough and fast primary estimate of hemodynamic status, but many studies put B-lines in (semi) quantitative correlations with aforementioned values [10]. Regarding EVLW estimate, Picano et al. put comet-tail score (CTS) and semiquantitative EVLW assessment to correlation (Table 2, [7]). The examination was done by standard method with 28 screening windows, B-lines counted in each window separately and then added to obtain the CTS. The inter and intra-observer error rate with this method is $5.1 \%$ and $7.4 \%$, respectively [7].

Agricola and colleagues devised a simple definition of positive comet tail test as multiple bilateral comets seen over the whole lung surface and a negative test being a rare single comet, no comets at all, or comets confined to the last intercostal spaces. Further, these workers made a comparison to EVLW estimated by the PiCCO method. A negative test had $90 \%$ sensitivity, and $89 \%$ specificity for correct detection of EVLW under $500 \mathrm{ml}$, and a positive test had $90 \%$ sensitivity and $86 \%$ specificity for detecting EVLW above $500 \mathrm{ml}$. In comparison to PiCCO and X-ray radiography, LUS detects subclinical EVLW excess below the threshold that causes alveolar edema with $87 \%$ sensitivity and $89 \%$ specificity. These investigators also noticed that B-lines can be observed 3 days before the clinical manifestations of fat embolism. Both observations can be explained by the fact that the interstitial edema, which preceded alveolar edema, can be detected by LUS [7].

Another study using the Volpicelli et al. 4-quadrant approach demonstrated $85.7 \%$ sensitivity and $97.7 \%$ specificity in detecting lung edema in comparison to X-ray radiography. The examination with LUS was considered positive for edema if it had at least 3 comets per scan, diffusely positive scans with more than one scan per side with comets, and bilateral presence of comets [7].

\section{B-lines and lung density}

Baldi et al. [4] compared CTS with lung weight and density calculated from CT scans and found a strong correlation which was better for density than weight; better for right lung than left; and better for upper lobes than lower ones. As density depends on two parameters, weight and volume, LUS can detect conditions in which only one of those parameters change. For example, lung volume expansion after thoracocentesis does not change lung weight, but it results in a reduction of lung density. The opposite is observed in pneumothoraces. Lung weight could not explain or detect these conditions on its own. Better CTS correlation with the right than the left lung could be explained with better LUS analysis of the right lung, considering there is no heart to interfere with the examination [4]. In this study, CTS was a sum of B-lines in anterolateral lung regions by standard method, where a score of 5 or less was considered normal, especially if the comets were found above the diaphragm. When the CTS was found to be above 5, the case was considered pathological with sensitivity of $94 \%$ and specificity of $100 \%$. The question arising from the fact that LUS penetrates only to the superficial/peripheral lung regions is how it can be relevant in evaluating lungs in total. Baldi et al. proved that lung density in the sub-pleural regions doesn't significantly differ from the rest of the lung tissue and also, US beam penetrates deeper in edematous lung. EVLW can be correctly detected because higher values are usually caused by diffuse conditions that affect central, as well as peripheral lung zones. One patient in the study was not classified as having a pathological finding as he suffered with effusion and consolidation in the posterior regions, which are not scanned by the standard technique, but can be significant [4].

\section{B-lines and connective tissue disease-associated interstitial lung disease}

Interstitial lung pathology is a significant cause of morbidity and mortality in connective tissue diseases, although the pathogenesis has not been fully established. Early detection can improve outcomes because it allows therapy to begin as early as possible. The challenge for this patient cohort is serial scans are needed for diagnosis and follow-up of the lung pathology, with the risk of high radiation dose from high-resolution computed tomography (HRCT). Pulmonary function tests are also used but correlate poorly with the stage of fibrosis, especially in early stages of the disease. For this reason, LUS was considered for patient follow-up and a good correlation was found between the number of B-lines and a fibrosis pattern on HRCT [14].

Systemic sclerosis is a disease most commonly associated with interstitial lung disease, with up to $90 \%$ of patients exhibiting changes on HRCT. Numerous methods including various number of scanning sites have been examined, with descriptive or semi-quantitative correlations established between number of B-lines and the underlying interstitial pathology. Similar studies have been conducted in other connective tissue diseases, such as rheumatoid arthritis, Sjögren's syndrome, and anti-synthetase syndrome, and all showed promising results [14]. 
TA B L E 1. Correlation of A pattern, B pattern and LVEF with cut off values of PAOP and EVLW including sensitivity and specificity for each correlation.

$\begin{array}{lcccccc}\text { PAOP } & \text { EVLW } & \text { A pattern } & \text { B pattern } & \text { LVEF } & \text { Sensitivity } & \text { Specificity } \\ \leq 18 \mathrm{mmHg} & & + & - & / & 0.857 & 0.4 \\ \leq 18 \mathrm{mmHg} & & + & - & \text { Normal } & 1 & 0.727 \\ & \leq 10 \mathrm{ml} / \mathrm{kg} & + & - & / & 0.81 & 0.909\end{array}$

TA B L E 2. Correlation of CTS score, number of comet tail and EVLW-semiquantitative EVLW assessment.

\begin{tabular}{|lcc|} 
Score & Number of Comet Tails & EVLW \\
\hline 0 & $<5$ & No signs \\
1 & $5-15$ & Mild \\
\hline 2 & $15-30$ & Moderate \\
3 & $>30$ & Severe \\
\hline
\end{tabular}

Source: Summerfield DT, Johnson BD. Lung Ultrasound Comet Tails - Technique and Clinical Significance. In: Squeri A, editor. Hot Topics in Echocardiography. IntechOpen; 2013. p. 51-64. Table 2, Semiquantitive classification of the Comet Tail Score (CTS) as proposed by Picano and colleagues; p. 58. [7].

\section{B-lines and parameters of cardiac failure}

Marcelo et al. compared LUS evaluation with clinical estimate through coronary calcium scoring (CCS), Nt-proBNP (a strong neurohormonal predictor of heart failure prognosis) and $\mathrm{ECHO}$ (E/e' ratio and LV filling pressure) for evaluating decompensation in patients with systolic heart failure. There is no gold standard for evaluating decompensation, but multiple parameters are used in combination to enhance sensitivity, and the utility of LUS in aiding this evaluation is under investigation. B-lines were counted in 28 standard anterolateral windows and a cut off was made at 15 (optimal results were obtained at this cut off). Using that cut off, B-lines were significantly correlated with standard parameters of decompensation. Cut off for Nt-proBNP was equal or greater than $1000 \mathrm{pg} / \mathrm{ml}$, which is not a consensus, but morbidity and mortality were noticed to be higher above that value [15].

An acute pulmonary edema very important in everyday ICU practice is weaning-induced pulmonary oedema (WIPO), which develops during spontaneous breathing trials due to impaired cardiac loading conditions. WIPO is a common cause of ventilator weaning failure which can be easily detected by LUS and treated in a timely manner, thus increasing the chances of successful weaning [16].

Some investigations suggest that LUS is superior to X-ray radiography for detecting fluid overload with the sensitivity similar to the one of Nt-proBNP. However, caution must be taken considering that Nt-proBNP is a marker of primary vascular fluid overload and does not always correlate with interstitial lung edema [7].

Good correlation was also found between B-lines and E/e' ratio, which was expected, as the presence of comets implies more EVLW due to higher LV filling pressure. Both Nt-
proBNP and E/e' ratio identify hemodynamic congestion, a state preceding lung congestion which can be identified by comets at later timepoints [15].

\section{Limitations and open questions}

Although identification of a B-line 'pattern' has proved useful in various conditions, some doubt remains concerning the utility of counting of B-line numbers in routine clinical practice [17]. The most important reasons for criticism of the applicability of B-line number is that it is partly an operator-dependent sign. Determining the exact number of B-lines is a relatively difficult task and depends on the interpretation of the observer, especially in real time POCUS. Recently, we and the others have found that reproducibility between observers was only moderate and intra-observer variability in B-lines numbering was the same [18, 19]. Consequently, the use of software algorithms for B-lines counting is recommended. Additional limits are the impact of ultrasound transducer selection and reproducibility between different probes which was found to be poor. Therefore, future research protocols on lung artifacts should focus on the selection of optimal ultrasound transducers and on the role of software for automatic B-line counting.

\section{Conclusion}

B-lines are being meticulously studied for potential correlation with many acute and chronic heart and lung conditions such as acute exacerbation of heart failure with lung edema, ARDS, interstitial pneumonia, pulmonary fibrosis, and pneumothorax. In pneumothorax, the lack of B-lines, along with the absence of a lung sliding sign, is used as a clear indication of air underneath the pleura, but caution must be taken in patients with bullous lung disease because the same underlying substrate can produce the same signs and thus resulting in a misleading diagnosis.

When B-lines are used with EVLW as a predictor of pulmonary congestion or in PAOP as an indicator of hemodynamic congestion, a good correlation is found between $\mathrm{B}$ lines and EVLW, but the estimation of PAOP is unreliable. However, when B-lines are combined with a rough LVEF estimation, PAOP prediction is improved. Higher sensitivity of LUS in comparison to X-ray radiography in detecting subclinical EVLW excess comes from the fact that LUS can detect interstitial edema which precedes the alveolar edema observed by X-ray radiography.

A good correlation was found between B-lines and lung weight and density, making LUS a simple and fast method for lung densitometry in the ICU. In the setting of cardiac failure, B-lines correlate well with hemodynamic congestion 
indicators such as Nt-proBNP and E/e' ratio.

In summary, lung ultrasound is becoming a valuable addition for diagnosis and guiding therapy. It has the advantage over older and more established diagnostic methods such as Xray radiography and $\mathrm{CT}$ since it is a fast and reliable tool with bedside availability that is safe from excess radiation exposure. This makes it an ideal tool in early patient assessment when more invasive monitoring methods are not available, especially when early application of adequate therapy and fluid resuscitation is crucial for patient management and evaluating patient prognosis. LUS is also invaluable in diagnosing emergency conditions, such as tension pneumothorax, which require immediate intervention.

\section{ACKNOWLEDGEMENTS}

I would like to express my gratitude to all those who helped me during the writing of this manuscript.

\section{CONFLICT OF INTEREST}

We declare that we do not have any commercial or associative interest that represents a conflict of interest in connection with the work submitted.

\section{REFERENCES}

[1] Gargani L, Volpicelli G. How i do it: Lung ultrasound. Cardiovasc Ultrasound. 2014;12:1-10.

[2] Ricci F, Aquilani R, Radico F, et al. Role and importance of ultrasound lung comets in acute cardiac care. Eur Hear journal Acute Cardiovasc care. 2015;4:103-112.

[3] Bianco F, Bucciarelli V, Ricci F, et al. Lung ultrasonography: A practical guide for cardiologists. J Cardiovasc Med. 2017;18:501-509.

[4] Baldi G, Gargani L, Abramo A, et al. Lung water assessment by lung ultrasonography in intensive care: a pilot study. Intensive Care Med. 2013;39:74-84.

[5] Enghard P, Rademacher S, Nee J, et al. Simplified lung ultrasound protocol shows excellent prediction of extravascular lung water in ventilated intensive care patients. Crit Care. 2015;19:36.
[6] Volpicelli G, Mussa A, Garofalo G, et al. Bedside lung ultrasound in the assessment of alveolar-interstitial syndrome. Am J Emerg Med. 2006;24:689-696.

[7] Summerfield DT, Johnson BD. Lung Ultrasound Comet Tails - Technique and Clinical Significance. In: Squeri A, editor. Hot Topics in Echocardiography. IntechOpen; 2013. p. 51-64.

[8] Jambrik Z, Monti S, Coppola V, et al. Usefulness of ultrasound lung comets as a nonradiologic sign of extravascular lung water. Am J Cardiol. 2004;93:1265-1270.

[9] Aziz SG, Patel BB, Ie SR, et al. The lung point sign, not pathognomonic of a pneumothorax. Ultrasound Q. 2016;32:277-279.

[10] Volpicelli G, Skurzak S, Boero E, et al. Lung Ultrasound Predicts Well Extravascular Lung Water but Is of Limited Usefulness in the Prediction of Wedge Pressure. Anesthesiology. 2014;121:320-327.

[11] Raheja R, Brahmavar M, Joshi D, et al. Application of Lung Ultrasound in Critical Care Setting: a Review. Cureus. 2019;11:e5233.

[12] Cox EGM, Wiersema R, Wong A, et al. Six versus eight and twentyeight scan sites for B-line assessment: differences in examination time and findings. Intensive Care Med. 2020;46:1063-1064.

[13] Šustić M, Šustić A. Unrecognized B line mimicked pneumothorax on M mode ultrasound. Signa Vitae. 2018;14:92.

[14] Wang YK, Gargani L, Barskova T, et al. Usefulness of lung ultrasound B-lines in connective tissue disease-associated interstitial lung disease: A literature review. Arthritis Res Ther. 2017;19:1-9.

[15] Miglioranza MH, Gargani L, Sant'Anna RT, et al. Lung Ultrasound for the Evaluation of Pulmonary Congestion in Outpatients. JACC Cardiovasc Imaging. 2013;6:1141-1151.

[16] Ferré A, Guillot M, Lichtenstein D, et al. Lung ultrasound allows the diagnosis of weaning-induced pulmonary oedema. Intensive Care Med. 2019;45:601-608.

[17] Sperandeo M, Trovato GM, Catalano D. Quantifying B-Lines on Lung Sonography: Insufficient Evidence as an Objective, Constructive, and Educational Tool. J Ultrasound Med. 2014;33:362.

[18] Haaksma ME, Smit JM, Heldeweg MLA, et al. Lung ultrasound and Blines: B careful! Intensive Care Med. 2020;46:544-545.

[19] Pičuljan A, Šustić M, Brumini G, et al. Reliability of B-line quantification by different-level observers and a software algorithm using point-of-care lung ultrasound. J Clin Monit Comput. 2019. doi: 10.1007/s10877-01900440-7.

How to cite this article: Ana Pičuljan, Alen Protić, Mehmed Haznadar, Alan Šustić. The Role of B-Line Artifacts on Lung U1trasound in Critically Ill Patients. Signa Vitae. 2020;16(2):1-7. doi:10.22514/sv.2020.16.0041. 